$\begin{array}{ll}\text { hep-th/9909013 } & \text { SU-ITP 99-40 }\end{array}$

\title{
Wilson loops as Precursors
}

\author{
Leonard Susskind and Nicolaos Toumbas \\ Department of Physics, Stanford University, \\ Stanford CA 94305-4060
}

\begin{abstract}
There is substantial evidence that string theory on $A d S_{5} \times S_{5}$ is a holographic theory in which the number of degrees of freedom scales as the area of the boundary in Planck units. Precisely how the theory can describe bulk physics using only surface degrees of freedom is not well understood. A particularly paradoxical situation involves an event deep in the interior of the bulk space. The event must be recorded in the (Schroedinger Picture) state vector of the boundary theory long before a signal, such as a gravitational wave, can propagate from the event to the boundary. In a previous paper with Polchinski, we argued that the "precursor" operators which carry information stored in the wave during the time when it vanishes in a neighborhood of the boundary are necessarily non-local. In this paper we argue that the precursors cannot be products of local gauge invariant operators such as the energy momentum tensor. In fact gauge theories have a class of intrinsically non-local operators which cannot be built from local gauge invariant objects. These are the Wilson loops. We show that the precursors can be identified with Wilson loops whose spatial size is dictated by the UV-IR connection.
\end{abstract}

August 1999

\section{Introduction}

This paper is concerned with the mechanism by which a holographic boundary theory can describe bulk physics. As emphasized in [1] [河] [3] a holographic 
description entails a vast reduction of the number of degrees of freedom needed to describe a region of bulk spacetime. Despite the large amount of circumstantial evidence for the holographic principle it is still very mysterious how such a sparse set of degrees of freedom can describe all local bulk physics. A particular challenge is to understand how events deep in the interior of the bulk space are recorded in the instantaneous (Schroedinger Picture) state vector of the boundary theory long before a signal can propagate from the event to the boundary 四.

Let us consider an example. For definiteness we will consider the $3+1$ dimensional super Yang Mills description of bulk physics in 5 dimensional AdS space [ [5] [6] [7]. We will be interested in the limit of large radius of curvature compared to the string scale. In this limit stringy excitations are negligible and the low energy supergravity approximation to bulk physics is reliable. On the SYM side we must take $N$ large keeping the 't Hooft coupling constant $g^{2} N$ fixed and large.

Suppose as in [4] an event takes place at the center 7 of AdS which radiates a gravitational wave toward the boundary. No signal including the wave itself can arrive at the boundary until a certain time elapses. If the original event is well localized near the center of a very large AdS space, the original bulk fields will typically be very spherically symmetric and time independent. In fact the only bulk field of importance is the time-time component of the metric whose behavior near the boundary records the presence of a certain amount of energy in the interior. On the SYM side this means that the energy momentum tensor is almost exactly homogeneous and consists of an homogeneous energy density and the pressure needed to make $\left\langle T_{\mu}^{\mu}\right\rangle=0$. However, this effect is featureless and provides no information about the profile of the gravitational wave. In addition, it is vanishingly small in the large $N$ limit since corrections to the metric due to the energy of the wave are smaller than the wave itself by factors of $\sqrt{G_{5}} \sim N^{-1}$. We refer the reader in [4] for notations and conventions. Thus, within a neighborhood of the boundary, all supergravity field functionals retain their original, vacuumlike expectation values, at least until light has had a chance to propagate from $r \sim 0$ to the boundary. The implication for the SYM theory is that all expectation values of local gauge invariant operators corresponding to the bulk fields, as well as expectation values of products of such operators, should initially be identical to their vacuum values and contain no information about the propagating wave.

This situation continues until the outgoing wave arrives at the boundary. At that time the perturbation on the boundary becomes nonzero and begins

\footnotetext{
${ }^{1}$ The usual $S_{5}$ factor in the correspondence plays no role in the present paper and will be ignored.

${ }^{2}$ Since AdS is a homogeneous space it has no preferred points. Center here means the origin of cavity coordinates.
} 
oscillating over the whole 3-sphere. From the SYM point of view, the energy momentum tensor and its products suddenly begin to coherently oscillate. The features of the gravitational wave can then be recovered from expectation values of the energy momentum tensor and its products.

Thus during the time when the wave vanishes within a neighborhood of the boundary, the SYM theory is excited to a non-vacuum-like state which we cannot distinguish from the vacuum by taking expectation values of local

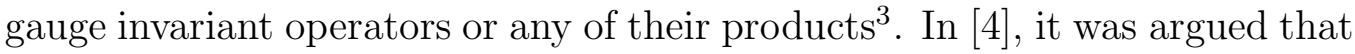
the holographic boundary theory must contain special non-local operators, called precursors, that distinguish such states and code in detail local bulk information. The precursors should become increasingly non-local the further the corresponding bulk process is from the boundary in accordance with the UV-IR connection [3]. In those cases in which the boundary theory has a gauge symmetry, the precursors must also be gauge invariant since they contain physical information. In the case of $\mathcal{N}=4 \mathrm{SYM}$ theory, this suggests that the precursors are Wilson loops whose size is dictated by the UV-IR relation.

We remark that there exists a rich class of generalized, equal-time Wilson loops being candidates for the precursors. Apart from conventional spatial Wilson loops, we may consider spatial Wilson loops with insertions of local gauge covariant operators. For example, we can consider the operator

$$
\operatorname{Tr} P F_{\mu \nu}\left(x_{1}\right) F^{\mu \nu}\left(x_{2}\right) W
$$

where $W$ is a Wilson loop passing through the points $x_{1}$ and $x_{2}$ and $P$ denotes path ordering. Presumably, such operators and their products form a complete set of observables in the boundary theory.

In [4], it was shown how a plane gravitational wave can be modeled by "squeezed" states constructed in free field theory. In particular, it was shown how to account for the oscillating energy density and the apparent acausality in the behavior of the energy momentum tensor required by the correspondence. It was found that this behavior is consistent with bounds required by general principles of quantum mechanics. In addition, apart from possible numerical coefficients, the free field theory model reproduces corrections to the linearized solution induced by non-linear terms in Einstein's equations involving the energy density of the wave.

In this note we model bulk waves with "squeezed states" constructed in the interacting SYM theory. We compute expectation values of local gauge invariant operators in the "squeezed states" and match them with the boundary data of the wave. We show that expectation values of products of local gauge invariant operators contain no additional information about

\footnotetext{
${ }^{3}$ We are assuming that local gauge invariant operators are in one-to-one correspondence with local observables of the bulk theory evaluated near the boundary.
} 
the profile of the wave in agreement with bulk causality. Our computations are done in the 't Hooft limit keeping only the leading terms in the $1 / N$ expansion. Finally, using the corespondence, we calculate expectation values of Wilson loops in the "squeezed states" and show how they carry non-trivial information if their size is as dictated by the UV-IR connection. We discuss the implications of our results for holography at the end.

Before concluding the introduction we will review some facts and conventions about the AdS-CFT correspondence. The metric of AdS in cavity coordinates is

$$
\begin{aligned}
d s^{2} & =R^{2}\left[\frac{\left(1+r^{2}\right)^{2}}{\left(1-r^{2}\right)^{2}} d t^{2}-\frac{4}{\left(1-r^{2}\right)^{2}}\left(d r^{2}+r^{2} d \Omega^{2}\right)\right] \\
& =R^{2} d S^{2}
\end{aligned}
$$

where the coordinates and $d S^{2}$ are dimensionless and $d \Omega^{2}$ is the metric of the unit 3-sphere. The center of AdS means the point $r=0$. Near a point of the boundary at $r=1$ the metric has the form

$$
d s^{2}=R^{2}\left[\frac{1}{z^{2}}\left(d t^{2}-d z^{2}-d x^{i} d x^{i}\right)\right]
$$

where $z=1-r$ and $x^{1}, x^{2}, x^{3}$ replace the coordinates of the 3 -sphere. For our purposes the metric (1.3) is to be regarded as a local approximation to the cavity metric. It is true, but irrelevant to our purposes, that the same metric also gives an exact description of a patch of AdS space. In any case we will call these the half-plane coordinates. The two dimensionless parameters $R / l_{s}$ and $g_{s}$ of the bulk theory $-l_{s}$ is the string length scale - are related to the SYM quantities $N$ and $g$ by

$$
\begin{aligned}
R / l_{s} & =\left(g^{2} N\right)^{1 / 4} \\
g_{s} & =g^{2} .
\end{aligned}
$$

The 5 and 10 dimensional Newton constants are given by

$$
\begin{aligned}
G_{5} & =G_{10} / R^{5} \\
G_{10} & =g_{s}^{2} l_{s}^{8} .
\end{aligned}
$$

We set $R=1$ for simplicity. The string length scale is then given by $l_{s}=$ $1 /\left(g^{2} N\right)^{1 / 4}$. Throughout we neglect numerical factors of order unity.

\section{Bulk Waves}

As in [4], we model bulk waves with "squeezed states" in the boundary theory. Our goal is to study expectation values of various operators in the "squeezed states" and identify the precursors that store local bulk information. 
For definiteness, let us consider a gravitational wave propagating radially outward from $r \sim 0$. In the next section, we will be interested in the case of a dilaton wave. Assume that the wave is in one of the lowest spherical harmonics on the 3-sphere. In half-plane coordinates the plane fronted wave has the form

$$
\gamma_{\mu \nu}(z, x, t)=\xi_{\mu \nu} \sqrt{G_{5}} \frac{\Phi(z, t)}{z^{2}}
$$

where $\gamma_{\mu \nu}(z, x, t)$ is defined by

$$
d s^{2}=\left[\frac{1}{z^{2}}\left(d t^{2}-d z^{2}-d x^{i} d x^{i}\right)\right]+\gamma_{\mu \nu}(z, x, t) d x^{\mu} d x^{\nu}
$$

and $\xi_{\mu \nu}$ is a transverse traceless polarization tensor with non-vanishing components in the $x$ directions. The polarization tensor is assumed normalized to unity.

Far away from the original sources, $\Phi(z, t)$ satisfies the same wave equation as a free, minimally coupled, massless scalar field in AdS. We use normalization conventions so that $\Phi(z, t)$ is canonically normalized. Thus we keep the amplitude $|\Phi(z, t)|$ finite, independent of $\mathrm{N}$, and the energy of the wave is finite. The corresponding operator in the SYM theory is $\xi_{i j} T_{i j} / N$. The 2-point function of this operator is of order $N^{0}$. Non-linear terms in the gravitational field equations are suppressed by additional factors of $\sqrt{G_{5}} \sim N^{-1}$ and will be ignored in this paper f

Near the boundary, normalizable solutions to the wave equation behave as follows

$$
\Phi(z, t) \sim z^{4} \int d \omega|\omega|^{3} \phi(\omega) e^{-i \omega t},
$$

with $\phi(\omega)=\phi^{*}(-\omega)$ since the field is real. According to the AdS-CFT correspondence, the wave makes a contribution to the SYM energy momentum tensor given by [1]

$$
\left\langle\frac{T_{i j}}{N}\right\rangle \sim-\left.\xi_{i j} z^{-4} \Phi(z, t)\right|_{z=0}
$$

We are interested in describing a wave emitted at a particular time $t_{0}$ in the past, near $r \sim 0$, so that, when $t<0$, the perturbation vanishes within a neighborhood of the boundary. This can be achieved if we choose the function $|\omega|^{3} \phi(\omega)$ to be analytic in the upper half $\omega$-plane and have the right asymptotic behavior as $\omega \rightarrow i \infty$. Then the boundary data vanish for $t<0$ and so does the contribution to $\langle T / N\rangle$. In general, the boundary data will be non-vanishing when $t>0$ since $\phi(\omega)$ will have singularities

${ }^{4}$ In $\llbracket$, these effects were included and it was shown how they can be reproduced in free field theory up to possible numerical coefficients. In the large $\mathrm{N}$ limit they are suppressed. However, they are important to recover consistency in the behavior of the energy momentum tensor required by general principles of quantum mechanics. We refer the reader in refs. [4] for a discussion of this point. 
in the lower half-plane. Also, causality of the bulk theory insures that the function $\Phi(z, t)$ describes a wave which, at any $t_{0}<t<0$, exactly vanishes for $z<|t|$. In addition, bulk causality requires that all local bulk fields evaluated in a neighborhood of the boundary, as well as products of such fields, retain their vacuum expectation values until $t=0$. Therefore, on the SYM side, expectation values of local gauge invariant operators and their products must be identical to their vacuum values until $t=0$.

\section{Squeezed States in Yang-Mills Theory}

We propose that during the propagation of the wave, the SYM theory is excited in the "squeezed state" defined by

$$
|\Psi\rangle=\exp \left[\frac{i \xi_{i j}}{N} \int d^{3} \vec{x} d t f(t) T_{i j}(\vec{x}, t)\right]|\Omega\rangle,
$$

where $|\Omega\rangle$ is the vacuum of the interacting theory and $f(t)$ is some real function related to the boundary data of the wave. The polarization $\xi_{i j}$ is taken to be traceless and symmetric. It will turn out to be the polarization of the wave. The state thus defined is unit-normalized 9 .

Our motivation in writing $\mathrm{Eq}(2.5)$ is as follows. In the large $\mathrm{N}$ limit with the 't Hooft coupling held fixed and large, $|\Psi\rangle$ corresponds to a coherent state in the bulk. To see this note that if we Fourier expand any local gauge invariant operator $O(\vec{x}, t)$

$$
O(\vec{x}, t)=\int_{\omega>0} d \omega d^{3} \vec{k} \mathcal{O}(\omega, \vec{k}) e^{-i \omega t+i \vec{x} \cdot \vec{k}}+\text { h.c. },
$$

then, to leading order in $1 / N$, the positive frequency modes $\mathcal{O}(\omega, \vec{k})$ behave like annihilation operators and the negative frequency modes $\mathcal{O}^{\dagger}(\omega, \vec{k})$ behave like creation operators [13]. In particular, their commutator is a c-number function. Thus, up to some irrelevant normalization factor, the state $|\Psi\rangle$ takes the form

$$
|\Psi\rangle \sim \exp \left[\int d \omega f(\omega) \mathcal{O}^{\dagger}(\omega, 0)\right]|\Omega\rangle .
$$

We see that if we identify the SYM vacuum with the bulk vacuum and the modes $\mathcal{O}(\omega, \vec{k})$ with the Fourier modes of the bulk field corresponding to $O$, $|\Psi\rangle$ becomes a coherent bulk state. This can always be done in the limit we are considering since in this limit, the relation

$$
O(x)=\left.z^{-4} \Phi(z, x)\right|_{z \rightarrow 0}
$$

holds as an exact operator relation [12] 13]. Coherent states in the bulk describe classical waves.

\footnotetext{
${ }^{5}$ In the free theory, to leading order in $1 / N$, it reduces to the "squeezed state" considered in [4, up to some normalization factor. Also, the energy momentum tensor is normalordered so that the vacuum energy density is zero.
} 
Next we calculate the expectation value

$$
\left\langle\Psi\left|\frac{T_{i j}(\vec{y}, \tau)}{N}\right| \Psi\right\rangle=\frac{1}{N}\left\langle\Omega\left|e^{-\frac{i \xi_{m n}}{N} \int f(t) T_{m n}(x)} T_{i j}(y) e^{\frac{i \xi m n}{N} \int f(t) T_{m n}(x)}\right| \Omega\right\rangle .
$$

In the 't Hooft limit and to leading order in $1 / N$, the commutator $\left[T_{i j}(y), T_{m n}(x)\right]$ is a $\mathrm{c}$-number function proportional to the central charge of the theory. Therefore, it is of order $N^{2}$. In fact, it is independent of the 't Hooft coupling and can be calculated in the free theory. This function vanishes both inside and outside the light-cone; it receives contributions only when the points $x$ and $y$ are at light-like separation. Hence, we can commute $T(y)$ past the exponential picking a factor proportional to this commutator. Recall also that the energy momentum tensor has zero expectation value in the vacuum. Then, to leading order in $1 / N$, the expectation value is given by

$$
\left\langle\Psi\left|\frac{T_{i j}(\vec{y}, \tau)}{N}\right| \Psi\right\rangle=\frac{i \xi_{m n}}{N^{2}} \int d t d^{3} \vec{x} f(t)\left[T_{i j}(\vec{y}, \tau), T_{m n}(\vec{x}, t)\right]
$$

The commutator is determined by the imaginary part of the time-ordered 2-point function of the energy momentum tensor, and so

$$
\left\langle\Psi\left|\frac{T_{i j}(\vec{y}, \tau)}{N}\right| \Psi\right\rangle=-\xi_{m n} \frac{2}{N^{2}} \int d t f(t) \epsilon(\tau-t) \operatorname{Im} \int d^{3} \vec{x}\left\langle T_{i j}(\vec{y}, \tau) T_{m n}(\vec{x}, t)\right\rangle+O\left(\frac{1}{N}\right) .
$$

The expectation value inside the integral is in the vacuum. All other components of the energy momentum tensor have expectation values of order $1 / N$ in this state.

The details of the calculation can be found in the appendix. Here, we write down the results. The spatial integral is imaginary and independent of $\vec{y}$. Simple dimensional analysis shows that it behaves like

$$
\frac{1}{|\tau-t|^{5}}
$$

Thus the expectation value of the energy momentum tensor in the "squeezed state" is given by

$$
\left\langle\Psi\left|\frac{T_{i j}(\vec{y}, \tau)}{N}\right| \Psi\right\rangle \sim \xi_{i j} \int d t f(t) \frac{1}{(\tau-t)^{5}} .
$$

If we Fourier transform $f(t)$

$$
f(t)=\int d \omega \frac{f(\omega)}{\omega} e^{-i \omega t}
$$

with $f(\omega)=-f^{*}(-\omega)$, we obtain

$$
\left\langle\Psi\left|\frac{T_{i j}(\vec{y}, \tau)}{N}\right| \Psi\right\rangle \sim i \xi_{i j} \int d \omega f(\omega)|\omega|^{3} e^{-i \omega \tau} .
$$


Comparing with $\mathrm{Eq}(2.3)$, we must set

$$
\phi(\omega) \sim i f(\omega)
$$

Note that we have chosen $\phi(\omega)$ so that the boundary data vanish for $t<0$. This does not imply that $f(t)$ is zero for $t<0$.

Finally, consider expectation values of products of the energy momentum tensor. Using the same method as before, we can easily see that these will differ from their vacuum values only by products of commutators. Schematically, we have

$$
\frac{1}{N^{2}}\left\langle\Psi\left|T_{1} T_{2}\right| \Psi\right\rangle=\frac{1}{N^{2}}\left\langle\Omega\left|T_{1} T_{2}\right| \Omega\right\rangle-\frac{1}{N^{2}} \int\left[T_{2}, T\right] \int\left[T_{1}, T\right] .
$$

The non-trivial component is just the product $\left\langle\Psi\left|T_{1}\right| \Psi\right\rangle\left\langle\Psi\left|T_{2}\right| \Psi\right\rangle$. Therefore, for $t_{1}, t_{2}<0$, the expectation value is identical to its vacuum value since each factor vanishes by construction. In any case, products of local gauge invariant operators contain no additional information about the profile of the wave. This is of course a consequence of large $N$ factorization.

\section{Wilson Loops}

In this section we show how the expectation value of a Wilson loop in a squeezed state carries non-trivial information about a dilaton wave. Since we are interested in the instantaneous state vector, a Wilson loop will typically mean a spatial loop with no extension in the time direction. To model a dilaton wave in the SYM theory, we replace $\xi_{i j} T_{i j} / N$ with $O=\operatorname{Tr} F^{2} / N$ in $\operatorname{Eq}(2.5)$.

We consider a conventional Wilson loop

$$
W(\mathcal{C})=\operatorname{Tr} P e^{i \oint A_{\mu} d x^{\mu}}
$$

for simplicity. In the 't Hooft limit and large 't Hooft coupling, the vacuum expectation value of this loop can be obtained from the area of a minimal world-sheet in AdS that ends on the loop at the boundary [8]. We consider a spatial Wilson loop evaluated at $\tau<0$ and oriented in the $x_{1}-x_{2}$ plane. We take the loop to be circular with size $a$. We regularize the VEV of this loop by dividing the divergent term proportional to the circumference.

We wish to calculate the expectation value $\langle\Psi|W| \Psi\rangle$ in the case when $f$ is small. In this case, we can expand the exponential keeping only linear terms in $f$. We do not expect higher order terms to modify our conclusions significantly, since in the 't Hooft limit their expectation values should factorize into products involving the linear term together with featureless (independent of $\tau$ and $a$ ) factors such as the VEV of products of $O$. 
Then the expectation value reduces to the following expression involving the commutator of the loop with the operator $O$

$$
\langle\Psi|W| \Psi\rangle=\langle W\rangle+i \int d t d^{3} \vec{x} f(t)\langle[W(\tau), O(\vec{x}, t)]\rangle .
$$

All expectation values in the RHS of the equation are vacuum expectation values. The first term is irrelevant to us since, by conformal invariance, it should be independent of the size of the loop $a$ (and $\tau$ ). In terms of timeordered vacuum expectation values the second term takes the form

$$
i \int d t d^{3} \vec{x} f(t) \epsilon(\tau-t)\left[\langle W(\tau) O(\vec{x}, t)\rangle-\left\langle W^{\dagger}(\tau) O(\vec{x}, t)\right\rangle^{*}\right] .
$$

The hermitian conjugate of the loop operator is obtained by reversing the orientation of the loop in the $x_{1}-x_{2}$ plane.

The Euclidean version of the "2-point functions" appearing in Eq(3.3) has been computed in [9] using the correspondence. One first finds a minimal world-sheet with the loop as its boundary. The world-sheet in turn induces a source term in the dilaton field equations through the coupling

$$
\frac{1}{2 \pi \alpha^{\prime}} \int d^{2} \sigma \sqrt{h} e^{\frac{\phi}{2}} .
$$

Here, $h_{i j}$ is the metric induced on the world-sheet when the background metric is in the Einstein frame. The term in the world-sheet action involving the curvature of the world-sheet is suppressed when the 't Hooft coupling is large and can be ignored. The 2-point function is given by the boundary data of the dilaton profile obtained by solving the classical field equations in the presence of the source. It depends only on two parameters, which are the polar co-ordinate of the operator $O$ on the plane defined by the loop $r$ and its perpendicular distance from the plane of the loop $y=\sqrt{(t-\tau)^{2}+x_{3}^{2}}$ [9]:

$$
\langle W(\tau) O(\vec{x}, t)\rangle \sim \frac{\langle W\rangle}{N} \frac{a^{4}}{\left[\left(y^{2}+r^{2}-a^{2}\right)^{2}+4 a^{2} y^{2}\right]^{2}} .
$$

We see that the 2-point function behaves like $1 / d^{4}$ when the operator approaches the loop, where $d=\sqrt{y^{2}+(r-a)^{2}}$ is the distance of the operator from the loop. To obtain the expression in Minkowski signature, we replace $(\tau-t)^{2} \rightarrow-(\tau-t)^{2}+i \epsilon$.

Before we continue with our calculation, we make some remarks about this correlation function. First, we see that it is of order $N^{0}$ since the expectation value of the loop itself is of order $N$. In fact, we may think of the operator $O=\operatorname{Tr} F^{2} / N$ as a small Wilson loop. The disconnected part of the 2-point function is zero since $O$ has vanishing VEV. The connected part of the 2point function receives contributions from world-sheets in the bulk that have 
the two loops as boundaries. The topology of these surfaces implies that the 2-point function is of order zero in the large $N$ expansion. Second, reversing the orientation of the loop does not change the result for the dilaton profile since the coupling of the world-sheet in the bulk to the dilaton field, $\mathrm{Eq}(3.4)$, remains the same. Hence, $\operatorname{Eq}(3.2)$ reduces to the following expression

$$
\langle\Psi|W| \Psi\rangle=\langle W\rangle-2 \int d t f(t) \epsilon(\tau-t) \operatorname{Im} \int d^{3} \vec{x}\langle W(\tau) O(\vec{x}, t)\rangle .
$$

First we do the spatial integral over the 2-point function and obtain the imaginary part as a function of the ratio

$$
\lambda=\frac{|\tau-t|}{a} .
$$

We also rescale $x_{3}$ and $r$ so that the variables of integration are dimensionless. In polar co-ordinates the integral takes the form

$$
I=\frac{2 \pi}{a} \int d r d x_{3} \frac{r}{\left[x_{3}^{2}-A^{2}+i \epsilon\right]^{2}\left[x_{3}^{2}-B^{2}+i \epsilon\right]^{2}},
$$

where

$$
A^{2}=\lambda^{2}-(r-1)^{2}
$$

and

$$
B^{2}=\lambda^{2}-(r+1)^{2} .
$$

The integrand has poles when $A^{2}$ and $B^{2}$ are positive. Therefore the integral has non-vanishing imaginary part. We explain the physical origin of these poles at the end of this section. We integrate over $x_{3}$ first, closing the contour from below and picking up the residues at the poles in the lower-half plane. Only non-negative real poles contribute to the imaginary part as a result of the $i \epsilon$ prescription.

In the appendix, we analyze the behavior of the imaginary part of the integral for three cases. When $\lambda \gg 1$, we find

$$
\operatorname{Im}(I) \sim \frac{1}{a \lambda^{5}}=\frac{a^{4}}{|\tau-t|^{5}}
$$

The result is identical to the result found in Eq.(2.12) for the case of local operators. This is of course the behavior one should expect to see. In this case, the temporal separation between the loop and the operator $\mathrm{O}$ is much bigger than the size of the loop, and we should be able to use the operator product expansion of the loop in terms of local gauge invariant operators to calculate the 2-point function. Note also that the 2-point function behaves like

$$
\langle W O\rangle \sim \frac{a^{4}}{\left[x^{2}-(\tau-t)^{2}\right]^{4}}
$$


when $\lambda \gg 1$, as the 2-point function of $O$ with itself. As $\lambda \rightarrow 1$, the imaginary part increases. When $\lambda \sim 1$, it is the biggest and behaves like

$$
\operatorname{Im}(I) \sim \frac{1}{a(\lambda-1)^{3 / 2}} .
$$

When $\lambda \ll 1$, we find that the imaginary part tends to zero like

$$
\operatorname{Im}(I) \sim \frac{\lambda^{2}}{a} .
$$

We can understand the result as follows. As explained below, in this case, the imaginary part of the integral receives contributions only when the operator is very close to the loop at $r \sim 1$ and $x_{3} \sim 0$. Their temporal separation is also small. Thus, using the Heisenberg equations of motion, we can approximate $O(\vec{x}, t)$ with

$$
O(\vec{x}, t)=O(\vec{x}, \tau)-\left.\partial_{t} O(\vec{x}, t)\right|_{t=\tau}(\tau-t) .
$$

We see that the operator $O$ commutes with the Wilson loop unless the two are in contact. Essentially, only a single point of the loop contributes to the commutator, a measure zero effect. The commutator in turn determines the imaginary part of the integral as we can see from $\mathrm{Eq}(3.2)$ and $\mathrm{Eq}(3.3)$. So we expect the imaginary part of the integral to vanish like a power of $\lambda^{2}$ or faster.

Let us now see how the expectation value of the Wilson loop in the "squeezed state"

$$
\langle\Psi|W(\tau)| \Psi\rangle=-2 \int d t f(t) \epsilon(\tau-t) \operatorname{Im}(I)
$$

carries information about the corresponding dilaton wave. The imaginary part of the integral is a function of $\lambda=|\tau-t| / a$. As before, we choose $f(t)$ so that $\langle\Psi|O(\tau)| \Psi\rangle$ exactly vanishes when $\tau<0$. At any $\tau<0$, the corresponding bulk wave vanishes for $z<|\tau|$. On the other hand, the expectation value of the Wilson loop has non-trivial time dependence when $\tau<0$. Early in the remote past, when $|\tau| \gg a$, we can approximate $\operatorname{Im}(I) \sim 1 /|\tau-t|^{5}$. Therefore, the expectation value tends to zero since it behaves exactly the same way as the expectation value of local gauge invariant operators given in $\operatorname{Eq}(2.13)$. When $|\tau| \ll a$, the imaginary part of $I$ is essentially independent of $\tau$ within most of the domain of integration but a small interval when $|t| \sim|\tau|$. Thus the expectation value receives its time-dependence from this small region of integration. Within this region though, $\lambda \ll 1$ and so the imaginary part of $I$ is tiny. Hence, the expectation value is featureless having essentially no time-dependence. When $|\tau| \sim a$, the expectation value receives non-trivial time-dependence due to competition effects between $f(t)$ and the imaginary part of $I$. It receives its biggest contribution from the region of integration near $t \sim 0$ since then $\lambda \sim 1$ and the imaginary part of 
$I$ diverges. When $|\tau| \sim a$, the wave is at co-ordinate distance $\sim a$ from the boundary. Thus the Wilson loop "detects" the wave when its distance from the boundary is comparable to the size of the loop, and reproduces details that depend on the profile of the wave. This is of course a manifestation of the UV-IR relation [3].

Another interesting example, is the case when $f(t)$ is oscillatory near $t=0$ and exponentially small otherwise. The oscillations are well concentrated near $t=0$. For example, we may take $f(t)$ to be a polynomial in $t$ times a gaussian. At any time $\tau$ other than zero, the corresponding bulk wave should be oscillatory near $z=|\tau|$ and very small in a neighborhood of the boundary. In this case, expectation values of local gauge invariant operators behave like

$$
\langle\Psi|O(\tau)| \Psi\rangle \sim \frac{f(0) \delta t}{|\tau|^{5}}
$$

and so they remain small unless the wave is at the boundary at $\tau=0$. Here, $\delta t$ is the characteristic decay time of the oscillations in $f(t)$. The expectation value of the Wilson loop though has very different time-dependence. Again, using the results for the behavior of the imaginary part of $I$ as a function of the ratio $|\tau| / a$, one can see that the expectation value is oscillatory when $|\tau| \sim a$ and suppressed when $|\tau| \gg a$ or $|\tau| \ll a$.

In short, when the wave is very close to the boundary, only small Wilson loops are excited. At that time, however, expectation values of local gauge invariant operators begin to oscillate. On the other hand, when the wave is far from the boundary only big Wilson are excited. This shows that the precursors are in fact Wilson loops.

Finally, let us try and understand the physical origin of the poles in the integrand in $\mathrm{Eq}(3.8)$. When the denominators vanish, the 2-point function has non-vanishing imaginary part since then the $i \epsilon$ prescription for treating the poles becomes relevant. As one can see from $\operatorname{Eq}(3.2)$ and $\operatorname{Eq}(3.3)$, the imaginary part of the 2-point function is determined by the vacuum expectation value of the commutator between the Wilson loop and the operator $O$. Therefore, at the poles the commutator is non-vanishing. Now, the commutator can be non-zero only when some part of the loop of non-trivial measure is on the light-cone of $O$. Then the commutator between the vector potential at any point whose separation from $O$ is light-like and $O$ is non-zero, and in turn all of them contribute to the commutator between the Wilson loop itself and $O$. This is precisely what happens at the poles as we show below. The imaginary part of the 2-point function vanishes when the loop is not intersecting the light-cone, and the contribution to the integral from this region of integration is real. Then, the 2-point function is non-singular as well.

Suppose the operator is at $t=0$. Then the loop can intersect with the past light-cone of $O$ only. For $\left|x_{3}\right|<\lambda$, the light-cone intersects the $x_{1}-x_{2}$ 
plane at a circle of radius

$$
\rho=\sqrt{\lambda^{2}-x_{3}^{2}}
$$

The polar co-ordinate $r$ of $O$ is the distance of the center of this circle from the center of the loop. The point of the loop closest to the center of the lightcone circle is at distance $|r-1|$ from it, while the one that is the farthest is at distance $r+1$. Clearly, when $A^{2}$ is negative, the loop is outside the light-cone and so no contributions to the imaginary part of the integral arise from this region of integration for any $\lambda$.

When $A^{2}$ is positive the loop and the light-cone circle intersect. We may choose, however, $\left|x_{3}\right|=A$ so that the two circles are tangent to each other. This is precisely when the integrand is singular. When the two circles are tangent the set of points on the loop that are close enough to the light-cone is of bigger measure and we get a contribution to the commutator and a pole in the 2-point function. For $\lambda>1$ the light-cone circle is tangent to the loop from the outside. The opposite is true for $\lambda<1$. In this case, the light-cone circle becomes smaller and smaller as $\lambda \rightarrow 0$ and the effect ceases to be important.

For $\lambda \geq 1$, we can choose $x_{3}$ small enough so that $\rho$ is bigger than the radius of the loop. If $B^{2}$ is positive, then, for $\left|x_{3}\right|<B$, the loop is inside the light-cone. For $\left|x_{3}\right|=B$ the two circles are tangent and again we have a pole in the 2-point function.

For $\lambda \geq 1$, the 2-point function becomes even more singular when $A=B$ at $r=0$ and $\rho=1$. In this case, the whole loop is on the light-cone. Therefore, we should expect a big contribution to the imaginary part of the integral from the small $r$ region. We expect this effect to amplify when $\lambda \sim 1$ since then, $x_{3} \sim 0$, and the operator is closer to the plane of the loop. Note also that the 2-point function is as singular when $A$ or $B$ are zero and $x_{3}=0$. The two effects combine when $\lambda=1$. The 2-point function can be the most singular in this case and we expect the imaginary part of the integral to be the biggest.

\section{Discussion}

The main purpose of this paper is to identify the non-local precursor fields of the SYM boundary theory that record information about local processes occurring deep in the interior of the bulk AdS spacetime. Causality of the bulk theory requires that the precursors are intrinsically non-local. They are not simple products of local operators corresponding to the classical supergravity fields in the standard AdS-CFT dictionary. Correlation functions of such products essentially remain featureless until the signal from the event arrives at the boundary. Yet, as in [4], we argue that the precursors store the information long before the signal can propagate to the boundary. 
In this paper, we study a rather simple case involving the propagation of a classical bulk wave toward the boundary. It is shown that when the wave vanishes within a neighborhood of the boundary, products of local gauge invariant operators retain their vacuum expectation values, whereas Wilson loops are excited when their size is of the same order as the co-ordinate distance of the wave from the boundary. A detailed translation of all the configurations of the bulk theory to the SYM theory is not yet available, but, as in the example of the wave, we believe that the precursors will involve Wilson loops with size dictated by the UV-IR connection. The precise way Wilson loops would store information about complicated processes in the bulk is very difficult to see. In particular, it remains a challenge to understand what precursors describe small Schwarzschild black holes at the center of AdS, or what configurations of Wilson loops provide the signal that a black hole forms in a head-on collision of two very energetic gravitons [4]. However, Wilson loops and their products form a complete set of gauge invariant operators in the SYM theory. This means that at any time one should be able to recover all the information about the state of the theory from their expectation values and expectation values of their products.

Our analysis has been carried out in the 't Hooft limit where we keep the 't Hooft coupling fixed and large and take $N \rightarrow \infty$. In this limit the bulk theory is manifestly local as it is well described by linearized supergravity. We do not consider $1 / N$ corrections in this paper since they are too small. We think that their effect is to modify the original expectation values of local gauge invariant operators by featureless components that do not carry any interesting information about the details of the relevant bulk process. For example, in the case of the gravitational wave considered in section II, the next-to-leading order $1 / N$ corrections depend on the total energy in the bulk, which is constant, but not on the detailed profile of the wave. Any interesting effect of bulk interactions should be recovered from such expectation values only when the signal of the event arrives at the boundary.

We believe that the "squeezed states" constructed in the SYM theory continue to accurately describe gravitational waves including the effect of bulk interactions. Evidence for this was found in [4], where the success of the free field theory model considered was linked with the non-renormalization theorem for the 3-point function of the energy momentum tensor. It would be very interesting to study the exact description of a gravitational wave in the flat space limit considered in [15][16]. In this limit we take $N$ large and $g$ fixed. We also keep bulk energies fixed in string units. This means that we have to consider energies in the SYM theory that scale like $N^{1 / 4}$. In flat space, plane gravitational waves are exact solutions of the theory and do not receive any stringy corrections [17]. However, we do not have any computational control in the SYM theory in this limit apart from conjectured non-renormalization theorems for the 2-point and 3-point functions of chiral 
primaries.

What really distinguishes the precursors in the case of $\mathcal{N}=4$ SYM theory from other non-local observables in the theory is that Wilson loops cannot be expressed in terms of finite polynomials of local gauge invariant operators corresponding to the bulk fields. Gauge invariance equips the boundary holographic theory with this rich class of intrinsically non-local observables so that it can reproduce traces of bulk causality and locality. Thus gauge invariance is crucial in the way this particular local conformal theory describes bulk physics. It would be interesting to understand the precise nature of the precursors in other AdS-CFT dualities in which the CFT is not a conventional gauge theory. For example, the $A d S_{3}$ case. In some of these examples the CFT is obtained from a gauge theory through renormalization group flows; however there is no remnant of the original gauge symmetry at the fixed point. It is particularly challenging to find special non-local observables in these examples as well so as to understand better the holographic nature of gravity.

Acknowledgements We would like to thank Vijay Balasubramanian, Gary Felder, Jason Prince Kumar, Maxim Perelstein, Joseph Polchinski, Simon Ross, Steve Shenker and Eva Silverstein for useful discussions. This work was supported in part by NSF grant 9870115 and by the US Department of Energy under contract DE-AC03-76SF00515.

\section{Appendix A}

In this appendix, we show how to compute the expectation value of the energy momentum tensor in the "squeezed state" as given in $\operatorname{Eq}(2.11)$ :

$$
\left\langle\Psi\left|\frac{T_{i j}(\vec{y}, \tau)}{N}\right| \Psi\right\rangle=-\xi_{m n} \frac{2}{N^{2}} \int d t f(t) \epsilon(\tau-t) \operatorname{Im} \int d^{3} \vec{x}\left\langle T_{i j}(\vec{y}, \tau) T_{m n}(\vec{x}, t)\right\rangle .
$$

The 2-point function can be found in [6]. It is given by

$$
\left\langle T_{i j}(\vec{y}, \tau) T_{m n}(\vec{x}, t)\right\rangle \sim N^{2} X_{i j m n} \frac{1}{\left[(\vec{x}-\vec{y})^{2}-(\tau-t)^{2}+i \epsilon\right]^{2}},
$$

where we drop numerical factors of order unity. The tensor $X_{i j m n}$ involves terms with four derivatives with respect to $y$. The precise formula can be found in [6].

Next we calculate the integral

$$
\int d^{3} \vec{x} \frac{1}{\left[(\vec{x}-\vec{y})^{2}-(\tau-t)^{2}+i \epsilon\right]^{2}}
$$

\footnotetext{
${ }^{6}$ Some interesting issues concerning this particular case were recently studied in [14].
} 
and obtain its imaginary part. The integral is independent of $\vec{y}$. We can also scale $|\Delta t|=|\tau-t|$ out of the integral to obtain the following expression

$$
\frac{4 \pi I}{|\Delta t|}
$$

where

$$
I=\int_{0}^{\infty} d x \frac{x^{2}}{\left(x^{2}-1+i \epsilon\right)^{2}} .
$$

Using integration by parts we can simplify the integral as follows

$$
I=\frac{1}{4} \int_{-\infty}^{\infty} d x \frac{1}{\left(x^{2}-1+i \epsilon\right)} .
$$

This integral can be done by contour integration. We close the contour from below picking up the residue at the pole $x=1-i \epsilon / 2$. We find

$$
I=-\frac{i \pi}{4}
$$

Since the integral is independent of $\vec{y}$ only the term with four time-derivatives in $X_{i j m n}$ contributes to the expectation value. Then the expectation value reduces to the following expression

$$
\xi_{i j} \int d t f(t) \epsilon(\tau-t) \partial_{\tau}^{4}\left(\frac{1}{|\tau-t|}\right) .
$$

This is the same expression as equation $\mathrm{Eq}(2.13)$ in Section II.

\section{Appendix B}

In this appendix, we show how to compute the imaginary part of the integral

$$
I=\frac{2 \pi}{a} \int d x_{3} d r \frac{r}{\left[x_{3}^{2}-A^{2}+i \epsilon\right]^{2}\left[x_{3}^{2}-B^{2}+i \epsilon\right]^{2}},
$$

where $A^{2}=\lambda^{2}-(r-1)^{2}$ and $B^{2}=\lambda^{2}-(r+1)^{2}$ as defined in Section III. We first do the $x_{3}$-integration closing the contour from below and evaluating the residue at the poles. Only real poles contribute to the imaginary part of the integral. We study the cases $\lambda>1$ and $\lambda<1$ separately. For $\lambda>1, A^{2}$ is positive for $0<r<\lambda+1$ and $B^{2}$ is positive for $0<r<\lambda-1$. For $\lambda<1$, $A^{2}$ is positive for $-\lambda+1<r<\lambda+1$ while $B^{2}$ is negative for all values of $r$.

For $\lambda>1$, the imaginary part of $\mathrm{I}$ is obtained from the imaginary part of the following expression

$$
\begin{aligned}
& \frac{i \pi^{2}}{16 a} \int_{0}^{\lambda-1} d r \frac{1}{r}\left[\frac{1}{(B-i \delta)^{3}}-\frac{1}{r} \frac{1}{(B-i \delta)}\right] \\
& +\frac{i \pi^{2}}{16 a} \int_{0}^{\lambda+1} d r \frac{1}{r}\left[\frac{1}{(A-i \delta)^{3}}+\frac{1}{r} \frac{1}{(A-i \delta)}\right] .
\end{aligned}
$$


This expression is obtained after we calculate the residue at the poles

$$
x_{3}=A-i \delta
$$

and

$$
x_{3}=B-i \delta .
$$

Here, $\delta$ is a small number to be set to zero at the end of the calculation.

Let us obtain the imaginary part for the case when $\lambda \gg 1$ first. We show that it vanishes like $1 / \lambda^{5}$. We split the integrals into three pieces:

$$
\begin{aligned}
& I_{1}=\frac{i \pi^{2}}{16 a} \int_{0}^{1} d r \frac{1}{r}\left[\frac{1}{B^{3}}+\frac{1}{A^{3}}-\frac{1}{r}\left(\frac{1}{B}-\frac{1}{A}\right)\right], \\
& I_{2}=\frac{i \pi^{2}}{16 a} \int_{1}^{\lambda-1} d r \frac{1}{r}\left[\frac{1}{(B-i \delta)^{3}}-\frac{1}{r} \frac{1}{(B-i \delta)}\right],
\end{aligned}
$$

and

$$
I_{3}=\frac{i \pi^{2}}{16 a} \int_{1}^{\lambda+1} d r \frac{1}{r}\left[\frac{1}{(A-i \delta)^{3}}+\frac{1}{r} \frac{1}{(A-i \delta)}\right] .
$$

The integrand in $I_{1}$ looks singular at $r=0$, but in fact it behaves like $r^{0}$ as $r \rightarrow 0$. To see this, we Taylor expand $A$ and $B$ in powers of $r$ to obtain

$$
\frac{1}{B}-\frac{1}{A}=\frac{2 r}{\left(\lambda^{2}-1\right)^{3 / 2}}+O\left(\frac{r^{2}}{\left(\lambda^{2}-1\right)^{5 / 2}}\right),
$$

and

$$
\frac{1}{B^{3}}+\frac{1}{A^{3}}=\frac{2}{\left(\lambda^{2}-1\right)^{3 / 2}}+O\left(\frac{r}{\left(\lambda^{2}-1\right)^{5 / 2}}\right) .
$$

This can be done since $0<r<1$ and $\lambda \gg 1$. Thus the integrand is of order $1 / \lambda^{5}$, and, therefore,

$$
\operatorname{Im}\left(I_{1}\right) \sim \frac{1}{a \lambda^{5}} .
$$

Next, consider $I_{2}$. The integrand is singular at $r=\lambda-1$ when $B=0$, but as we will show the imaginary part is finite. The small number $\delta$ regulates the imaginary part. The imaginary part is given by

$$
\operatorname{Im}\left(I_{2}\right)=\frac{\pi^{2}}{16 a} \int_{1}^{\lambda-1} d r \frac{1}{r}\left[\frac{\left(B^{3}-3 \delta^{2} B\right)}{\left(B^{2}+\delta^{2}\right)^{3}}-\frac{1}{r} \frac{B}{\left(B^{2}+\delta^{2}\right)}\right] .
$$

Change variables by setting

$$
r+1=\sqrt{\lambda^{2}-x^{2}}
$$


to find

$$
\begin{aligned}
\operatorname{Im}\left(I_{2}\right)= & \frac{\pi^{2}}{16 a} \int_{0}^{\sqrt{\lambda^{2}-4}} d x\left\{\frac{1}{\left(\lambda^{2}-x^{2}\right)^{1 / 2}\left[\left(\lambda^{2}-x^{2}\right)^{1 / 2}-1\right]} \times\right. \\
& {\left.\left[\frac{\left(x^{4}-3 \delta^{2} x^{2}\right)}{\left(x^{2}+\delta^{2}\right)^{3}}-\frac{1}{\left[\left(\lambda^{2}-x^{2}\right)^{1 / 2}-1\right]} \frac{x^{2}}{\left(x^{2}+\delta^{2}\right)}\right]\right\} . }
\end{aligned}
$$

Similarly, if we change variables

$$
r-1=\sqrt{\lambda^{2}-x^{2}},
$$

the imaginary part of $I_{3}$ becomes

$$
\begin{aligned}
\operatorname{Im}\left(I_{3}\right)= & \frac{\pi^{2}}{16 a} \int_{0}^{\lambda} d x\left\{\frac{1}{\left(\lambda^{2}-x^{2}\right)^{1 / 2}\left[\left(\lambda^{2}-x^{2}\right)^{1 / 2}+1\right]} \times\right. \\
& {\left.\left[\frac{\left(x^{4}-3 \delta^{2} x^{2}\right)}{\left(x^{2}+\delta^{2}\right)^{3}}+\frac{1}{\left[\left(\lambda^{2}-x^{2}\right)^{1 / 2}+1\right]} \frac{x^{2}}{\left(x^{2}+\delta^{2}\right)}\right]\right\} . }
\end{aligned}
$$

Combining the two we are left with the following simpler integrals

$$
X_{1}=\int_{0}^{\sqrt{\lambda^{2}-4}} d x \frac{2}{\left(\lambda^{2}-1-x^{2}\right)}\left[\frac{\left(x^{4}-3 \delta^{2} x^{2}\right)}{\left(x^{2}+\delta^{2}\right)^{3}}-\frac{2}{\left(\lambda^{2}-1-x^{2}\right)} \frac{x^{2}}{\left(x^{2}+\delta^{2}\right)}\right]
$$

and

$$
X_{2}=\int_{\sqrt{\lambda^{2}-4}}^{\lambda} d x \frac{1}{\left(\lambda^{2}-x^{2}\right)^{1 / 2}\left[\left(\lambda^{2}-x^{2}\right)^{1 / 2}+1\right]}\left[\frac{1}{x^{2}}+\frac{1}{\left[\left(\lambda^{2}-x^{2}\right)^{1 / 2}+1\right]}\right] .
$$

In $X_{2}$ we drop the terms proportional to $\delta$ since the integrand is well behaved within the domain of integration. This integral can be obtained in terms of logarithms. We do not write the whole expression down. Rather, we write its series expansion in terms of powers of $1 / \lambda$ :

$$
X_{2}=\frac{2}{3 \lambda}+\frac{4}{3 \lambda^{3}}+\frac{2}{\lambda^{5}}+O\left(\frac{1}{\lambda^{7}}\right) .
$$

Next we calculate $X_{1}$. First choose a cut-off $\epsilon$ which we will take to be zero at the end. We must take the limit $\delta \rightarrow 0$ first. Then $X_{1}$ reduces to the following two pieces

$$
\int_{0}^{\epsilon} d x \frac{2}{\left(\lambda^{2}-1\right)}\left[\frac{\left(x^{4}-3 \delta^{2} x^{2}\right)}{\left(x^{2}+\delta^{2}\right)^{3}}\right]+O(\epsilon),
$$

and

$$
\int_{\epsilon}^{\sqrt{\lambda^{2}-4}} d x \frac{2}{\left(\lambda^{2}-1-x^{2}\right)}\left[\frac{1}{x^{2}}-\frac{2}{\left(\lambda^{2}-1-x^{2}\right)}\right]+O\left(\delta^{2}\right) .
$$


The first piece is given explicitly by

$$
\frac{-2 \epsilon^{3}}{\left(1-\lambda^{2}\right)\left(\delta^{2}+\epsilon^{2}\right)^{2}} .
$$

The second piece reduces to

$$
\frac{2}{\left(\lambda^{2}-1\right) \epsilon}-\frac{2}{3 \sqrt{\lambda^{2}-4}}+O(\epsilon) .
$$

We see that after taking the $\delta \rightarrow 0$ limit the singular term of order $1 / \epsilon$ cancels and we are left with a finite result. Taylor-expanding in powers of $1 / \lambda$ yields

$$
X_{1}=-\frac{2}{3 \lambda}-\frac{4}{3 \lambda^{3}}-\frac{4}{\lambda^{5}}+O\left(\frac{1}{\lambda^{7}}\right) .
$$

Adding the result to $X_{2}$ obtained in $\mathrm{Eq}(\mathrm{B} .18)$, we find

$$
\operatorname{Im}\left(I_{2}+I_{3}\right) \sim \frac{1}{a \lambda^{5}} .
$$

Since $I_{1} \sim 1 / \lambda^{5}$ as well, the imaginary part of $I$ decreases like $\lambda^{-5}$.

Then we study the case when $\lambda \sim 1^{+}$. We set $\lambda-1=e$ and obtain the imaginary part as a power series expansion in $e$. We show that the imaginary part behaves like $e^{-3 / 2}$. We study the imaginary part of each of the following integrals

$$
I_{2}=\frac{i \pi^{2}}{16 a} \int_{d}^{e} d r \frac{1}{r}\left[\frac{1}{(B-i \delta)^{3}}-\frac{1}{r} \frac{1}{(B-i \delta)}\right]
$$

and

$$
I_{3}=\frac{i \pi^{2}}{16 a} \int_{d}^{e+2} d r \frac{1}{r}\left[\frac{1}{(A-i \delta)^{3}}+\frac{1}{r} \frac{1}{(A-i \delta)}\right] .
$$

Here, $d$ is a small number that regulates each of the integrals near $r=0$. At the end, after taking $d \rightarrow 0$, the sum of $I_{2}$ and $I_{3}$ will turn out to be finite independent of $d$.

To evaluate the imaginary part of $I_{2}$, we do the same change of variables as before, $\mathrm{Eq}(\mathrm{B} .12)$, and obtain the same expression as $\mathrm{Eq}(\mathrm{B} .13)$ but now with the domain of integration being $0<x<\sqrt{2(e-d)+e^{2}}$. For $I_{3}$, however, we cannot use the same change of variables as in Eq.(B.14) within the whole domain of integration since, for $r<1, r-1$ is negative. When $r<1$, we must set

$$
1-r=\sqrt{\lambda^{2}-x^{2}} .
$$

Then the imaginary part of $I_{3}$ is given by $\mathrm{Eq}(\mathrm{B} .15)$ plus an additional term $\frac{\pi^{2}}{16 a} \int_{\sqrt{2(e+d)+e^{2}}}^{\lambda} d x \frac{1}{\left(\lambda^{2}-x^{2}\right)^{1 / 2}\left[-\left(\lambda^{2}-x^{2}\right)^{1 / 2}+1\right]}\left[\frac{1}{x^{2}}+\frac{1}{\left[-\left(\lambda^{2}-x^{2}\right)^{1 / 2}+1\right]}\right]$. 
Combining the three pieces together, one is left with the following integrals

$$
\begin{aligned}
& X_{1}=\int_{0}^{\sqrt{2(e-d)+e^{2}}} d x \frac{2}{\left(2 e+e^{2}-x^{2}\right)}\left[\frac{\left(x^{4}-3 \delta^{2} x^{2}\right)}{\left(x^{2}+\delta^{2}\right)^{3}}-\frac{2}{\left(2 e+e^{2}-x^{2}\right)} \frac{x^{2}}{\left(x^{2}+\delta^{2}\right)}\right], \\
& X_{2}=\int_{\sqrt{2(e-d)+e^{2}}}^{\sqrt{2(e+d)+e^{2}}} d x \frac{1}{\left(\lambda^{2}-x^{2}\right)^{1 / 2}\left[\left(\lambda^{2}-x^{2}\right)^{1 / 2}+1\right]}\left[\frac{1}{x^{2}}+\frac{1}{\left[\left(\lambda^{2}-x^{2}\right)^{1 / 2}+1\right]}\right],
\end{aligned}
$$

and

$$
X_{3}=\int_{\sqrt{2(e+d)+e^{2}}}^{1+e} d x \frac{2}{\left[(1+e)^{2}-x^{2}\right]^{1 / 2}\left(x^{2}-2 e-e^{2}\right)}\left[\frac{1}{x^{2}}+\frac{\left(2+2 e+e^{2}-x^{2}\right)}{\left(x^{2}-2 e-e^{2}\right)}\right] .
$$

First note that

$$
X_{2}=\int_{\sqrt{2(e-d)+e^{2}}}^{\sqrt{2(e+d)+e^{2}}} d x \frac{1}{x^{2}}+O(\sqrt{e}) .
$$

This in turn is of order $d$, and, therefore, it vanishes since we take $d \rightarrow 0$. The first integral can be calculated as before. The small number $\delta$ regulates the integral near the lower limit $x=0$, exactly the same way as before. We are left with

$$
X_{1}=-\frac{1}{\sqrt{e(2+e)} d}+\text { finite in } d .
$$

Here, the finite piece in $d$ is of order $e^{-3 / 2}$. The singular term of order $d^{-1}$ arises from the second piece of the integrand which diverges like

$$
\frac{1}{[\sqrt{e(2+e)}-x]^{2}}
$$

near the upper limit of integration. Finally, consider the integral $X_{3}$. This should be dominated by the singular terms near the lower limit of integration. Near the upper limit of integration the integrand behaves like $1 / \sqrt{1+e-x}$, but the integral converges. Therefore, we can expand in powers of $\left(x^{2}-2 e-\right.$ $e^{2}$ ) and consider only the singular terms. We find

$$
X_{3}=\int_{\sqrt{2(e+d)+e^{2}}}^{1+e} d x\left[\frac{1}{x^{2}}+\frac{2}{x^{2}\left(x^{2}-2 e-e^{2}\right)}+\frac{4}{\left(x^{2}-2 e-e^{2}\right)^{2}}\right]+\text { finite. }
$$

The finite piece is finite both in the $d \rightarrow 0$ and $e \rightarrow 0$ limits. We find that

$$
X_{3}=\frac{1}{\sqrt{e(2+e)} d}+\text { finite in } d,
$$


and so the singular term of order $d^{-1}$ cancels. Again, the piece finite in $d$ is of order $e^{-3 / 2}$. After taking the $d \rightarrow 0$ limit, we combine the finite piece in $X_{3}$ with the finite piece in $X_{1}$ and expand in powers of $e$, to find

$$
X_{1}+X_{3}=-\frac{1}{\sqrt{2} e^{3 / 2}}+\frac{7}{4 \sqrt{2 e}}+O\left(e^{0}\right)
$$

Thus the imaginary part of $I$ behaves like

$$
\operatorname{Im}(I) \sim-\frac{1}{a(\lambda-1)^{3 / 2}}
$$

We also note that the imaginary part is negative for $\lambda \sim 1^{+}$. This was also the case in the $\lambda \gg 1$ limit. Thus we expect the imaginary part to be increasing negatively as $\lambda \rightarrow 1$.

Finally, let us analyze the case when $\lambda \ll 1$. In this case $B^{2}$ is negative and does not contribute to the imaginary part. We have to extract the imaginary part from the following expression

$$
\frac{i \pi^{2}}{16 a} \int_{1-\lambda}^{1+\lambda} d r \frac{1}{r}\left[\frac{1}{(A-i \delta)^{3}}+\frac{1}{r} \frac{1}{(A-i \delta)}\right] .
$$

We choose to make the following change of variables first

$$
u=r-1 .
$$

The imaginary part is then given by

$$
\operatorname{Im}(I)=\frac{\pi^{2}}{16 a} \int_{-\lambda}^{\lambda} d u \frac{1}{u+1}\left[\frac{\left(A^{3}-3 \delta^{2} A\right)}{\left(A^{2}+\delta^{2}\right)^{3}}+\frac{1}{u+1} \frac{A}{\left(A^{2}+\delta^{2}\right)}\right],
$$

where $A^{2}=\lambda^{2}-u^{2}$. This in turn can be written as follows

$$
\frac{\pi^{2}}{8 a} \int_{0}^{\lambda} d u \frac{1}{1-u^{2}}\left[\frac{\left(A^{3}-3 \delta^{2} A\right)}{\left(A^{2}+\delta^{2}\right)^{3}}+\frac{1+u^{2}}{1-u^{2}} \frac{A}{\left(A^{2}+\delta^{2}\right)}\right] .
$$

Now change variables by setting $A^{2}=x^{2}$ to get

$$
\frac{\pi^{2}}{8 a} \int_{0}^{1} d x \frac{1}{\sqrt{1-x^{2}}\left(1-\lambda^{2}+\lambda^{2} x^{2}\right)}\left[\frac{\left(x^{4}-3 \delta^{2} x^{2}\right)}{\lambda^{2}\left(x^{2}+\delta^{2}\right)^{3}}+\frac{\left(1+\lambda^{2}-\lambda^{2} x^{2}\right)}{\left(1-\lambda^{2}+\lambda^{2} x^{2}\right)} \frac{x^{2}}{\left(x^{2}+\delta^{2}\right)}\right],
$$

where we have rescaled $\mathrm{x}$ with $1 / \lambda$. The second piece in the integral becomes

$$
\frac{\pi^{2}}{8 a} \int_{0}^{1} d x \frac{1}{\sqrt{1-x^{2}}}+O\left(\lambda^{2}\right)=\frac{\pi^{3}}{16 a}+O\left(\lambda^{2}\right)
$$


The first piece can be integrated using the same method as before. Choose a small cut-off $\epsilon$ and write the integral in terms of

$$
L_{1}=\frac{\pi^{2}}{8 a} \int_{0}^{\epsilon} d x \frac{\left(x^{4}-3 \delta^{2} x^{2}\right)}{\lambda^{2}\left(1-\lambda^{2}\right)\left(x^{2}+\delta^{2}\right)^{3}}+O(\epsilon)
$$

and

$$
L_{2}=\frac{\pi^{2}}{8 a} \int_{\epsilon}^{1} d x \frac{1}{\lambda^{2} x^{2} \sqrt{1-x^{2}}\left(1-\lambda^{2}+\lambda^{2} x^{2}\right)}+O\left(\delta^{2}\right) .
$$

Next we observe that

$$
L_{2}=\frac{\pi^{2}}{8 a} \int_{\epsilon}^{1} d x \frac{1}{\lambda^{2}\left(1-\lambda^{2}\right) x^{2} \sqrt{1-x^{2}}}-\frac{\pi^{2}}{8 a} \int_{0}^{1} d x \frac{1}{\sqrt{1-x^{2}}}+O\left(\lambda^{2}\right) .
$$

Evaluating $L_{1}$ and taking $\delta \rightarrow 0$, we are left with

$$
L_{1}=-\frac{\pi^{2}}{8 a \lambda^{2}\left(1-\lambda^{2}\right) \epsilon} .
$$

Similarly, after taking $\epsilon \rightarrow 0, L_{2}$ reduces to

$$
L_{2}=\frac{\pi^{2}}{8 a \lambda^{2}\left(1-\lambda^{2}\right) \epsilon}-\frac{\pi^{3}}{16 a}+O\left(\lambda^{2}\right)
$$

Adding the three pieces together, we see that the imaginary part tends to zero when $\lambda \ll 1$ like $\lambda^{2}$.

\section{References}

[1] G. 't Hooft, "Dimensional Reduction in Quantum Gravity," grqc/9310026.

[2] L. Susskind, "The World as a Hologram," hep-th/9409089.

[3] L. Susskind, Edward Witten, "The Holographic Bound in Anti-de Sitter Space," hep-th/9805114.

[4] Joseph Polchinski, Leonard Susskind, Nicolaos Toumbas, "Negative Energy, Superluminosity and Holography," hep-th/9903228

[5] Juan M. Maldacena, "The Large N Limit of Superconformal Field Theories and Supergravity," hep-th/9711200.

[6] S.S. Gubser, I.R. Klebanov, A.M. Polyakov, "Gauge Theory Correlators from Non-Critical String Theory," hep-th/9802109 
[7] Edward Witten, "Anti De Sitter Space And Holography," hepth/9802150.

[8] Juan M. Maldacena, "Wilson loops in large N field theories," hepth/9803002. S. J. Rey, J. Yee, "Macroscopic strings as heavy quarks in large N gauge theory and AdS supergravity," hep-th/9803001.

[9] David Berenstein, Richard Corrado, Willy Fischler, Juan M. Maldacena "The Operator Product Expansion for Wilson Loops and Surfaces in the large N limit," hep-th/9809188.

[10] L.H. Ford, Thomas A. Roman, "The Quantum Interest Conjecture," gr-qc/9901074.

[11] V. Balasubramanian, P. Kraus, "A Stress Tensor for Anti-de Sitter Gravity," hep-th/9902121. R. C. Myers, "Stress tensors and Casimir Energies in the AdS/CFT correspondence," hep-th/9903203.

[12] V. Balasubramanian, P. Kraus, A. Lawrence, and S. Trivedi "Holographic probes in Anti de Sitter Space-times," hep-th/9808017.

[13] T. Banks, M. R. Douglas, G. T. Horowitz, and E. Martinec, "AdS Dynamics from Conformal Field Theory," hep-th/9808016.

[14] V. Balasubramanian, S. Ross, "Holographic particle detection," hepth/9906226.

[15] Joseph Polchinski, "S-Matrices from AdS Spacetime," hep-th/9901076.

[16] Leonard Susskind, "Holography in the Flat Space Limit," hepth/9901079.

[17] R. Kallosh and A. Rajaraman, "Vacua of M-theory and String theory," hep-th/9805041. 